\title{
Role of Laparoscopy in Infertility : Review Article
}

\section{JAHAN S}

\begin{abstract}
Infertility is defined as failure to conceive during one year of unprotected frequent intercourse. Leading causes of infertility include tubal disease, ovulatory disorders, uterine or cervical factors, endometriosis and male factor infertility. A laparoscope is a thin fiber optic telescope that is inserted into the abdomen usually through the belly button. The fiber optics allow a light to used to see inside the abdomen. Carbon dioxide (CO2) gas is placed into the abdomen prior to inserting the laparoscope. Generally, laparoscopy should be reserved for couples who have already completed a more basic infertility evaluation including assessing for ovulation, ovarian reserve, ultrasound and hysterosalpingogram for the female and semen analysis for the male. Laparoscopy can help physicians diagnose many gynecological problems including endometriosis, uterine fibroids and other structural abnormalities, ovarian cysts, adhesions (scar tissue), and ectopic pregnancy. Robotic assisted laparoscopic surgery ( $R A L$ ) is a more recent development and a form of operative laparoscopy. In RAL, the instruments and telescope are very similar to conventional laparoscopy, but they are attached to
\end{abstract}

\section{Introduction}

Infertility is defined as failure to conceive during one year of unprotected frequent intercourse ${ }^{1}$. Leading causes of infertility include tubal disease, ovulatory disorders , uterine or cervical factors, endometriosis and male factor infertility ${ }^{1,2,3}$. Major causes according to WHO on a global basis are malnutrition, pelvic tuberculosis and puerperal infections leading to tubal blockage $^{4}$.

Laparoscopy is an essential step and a standard procedure in the investigation and evaluation of infertile females before initiating infertility treatment ${ }^{5,6}$. In the absence of clinical signs and symptoms suggestive of a diagnosis, laparoscopy offers an excellent means through direct visualization to elucidate the hidden pathology.

Dr. Samsad Jahan, Associate Professor, Department of Gynecology \& Obstetrics, BIRDEM, Dhaka, Bangladesh

Address of Correspondence: Dr. Samsad Jahan, Associate Professor, Department of Gynecology \& Obstetrics, BIRDEM, Dhaka, Bangladesh

Received: 12 May, 2012

Accepted: 21 June, 2012 a robot which in turn is controlled by the surgeon who is seated at a viewing console. Women who have been diagnosed with endometriosis are more likely to experience infertility, and observational studies have shown that the monthly probability of pregnancy in women with endometriosis is about half of the probability in normal women. In spite of this well-documented association, a true cause and effect relationship has not been established. Laparoscopy is used world-wide to investigate infertility. It is an essential part of full assessment and treatment of infertility. It provides direct visualization of the pelvic organs, ovarian and tubal status and can elucidate the site of tubal obstruction. It has got an advantage of direct visualization of the pelvic organs and the peri-tubal status resulting in greater information as compared to hysterosalpingography and ultrasonography. The advance in instrument technology has made this procedure more productive and less hazardous. Laparoscopy is the most dependable tool to investigate pelvic pathology. The role of laparoscopy in diagnosis of infertility both primary as well as secondary is established beyond any doubt.

(Birdem Med J 2012; 2(2): 99-103)

Diagnostic laparoscopy is generally accepted as the most accurate procedure to detect tubal pathology and endometriosis. Less invasive diagnostic tests such as patient's history, chlamydia antibody testing (CAT), ultrasonography and hysterosalpingography (HSG) are available, but it is still a matter of debate how the value of these tests compares with laparoscopy in the infertility work-up ${ }^{7}$ Several studies describe risk factors for tubal pathology such as previous abdominal surgery and previous pelvic inflammatory disease (PID). However, up to $68 \%$ of patients without any of these risk factors can still possess abnormalities as shown by laparoscopy $8,9,10$

Several studies describe the accuracy of CAT and HSG with diagnostic laparoscopy (DLS) as gold standard. A meta-analysis of studies comparing chlamydia antibody titres and laparoscopy for tubal patency and peritubal adhesions has shown that the discriminative capacity of chlamydia antibody titres in the diagnosis of any tubal pathology is comparable to that of HSG in the diagnosis of tubal occlusion ${ }^{11}$ Although CAT can be determined at low cost, it fails to provide information 
about the severity of tubal pathology, which is of importance to fertility prognosis and, subsequently, to infertility treatment. Furthermore, it cannot detect tubal pathology due to other causes or endometriosis.

A meta-analysis of 20 studies comparing HSG and laparoscopy for tubal patency and peritubal adhesions showed that HSG is of limited use for detecting tubal patency because of its low sensitivity, though its high specificity makes it a useful test for confirming the presence of tubal obstruction. For the evaluation of tubal patency and peritubal adhesions, but especially endometriosis, HSG is not reliable and requires laparoscopy ${ }^{12}$. Laparoscopy still reveals tubal pathology or endometriosis in 35-68\% of cases, even after normal HSG 12,14,15,16,17,18, 10

According to World Health Organization (WHO) guidelines, DLS is still recommended as a minimal requirement in the investigation of infertility in the female 19. However, it remains questionable whether DLS in general provides more information to further diagnosis and treatment decisions. There is a growing tendency to bypass diagnostic laparoscopy in couples with a normal HSG who will undergo intrauterine insemination (IUI) treatment for idiopathic infertility, mild male subfertility and cervical hostility.

Given the invasive and costly nature of the procedure, we considered it clinically relevant to investigate the effectiveness of the DLS as part of the IUI work-up. The purpose of the diagnostic laparoscopy is first to trace abnormalities and secondly to treat them when necessary. We questioned if the laparoscopy should always be performed before starting IUI. Considering treatment efficacy and applying cumulative pregnancy rate findings of the study by ${ }^{20}$, we expected that the difference in the cumulative pregnancy rate with and without laparoscopic treatment would be no more than $10 \%$ in the IUI setting. To demonstrate such a difference, a large study sample of at least 1000 patients would have been necessary.

\section{What is a laparoscope?}

A laparoscope is a thin fiber optic telescope that is inserted into the abdomen usually through the belly button. The fiber optics allow a light to used to see inside the abdomen. Carbon dioxide (CO2) gas is placed into the abdomen prior to inserting the laparoscope. This lifts the abdominal wall and allows for some separation of the organs inside the abdomen making it easier for the fertility doctor to see the reproductive organs during the surgery.

What are the advantages of laparoscopy for infertility?

Which infertile patients should have laparoscopy?

Generally, laparoscopy should be reserved for couples who have already completed a more basic infertility evaluation including assessing for ovulation, ovarian reserve, ultrasound and hysterosalpingogram for the female and semen analysis for the male. Some couples may elect to skip laparoscopy in favor of proceeding to other fertility treatments such as superovulation with fertility medications combined with intrauterine insemination or in vitro fertilization.

\section{Laparoscopy versus IVF}

Two commonly encountered problems during a laparoscopy, pelvic adhesions and endometriosis, can also be effectively treated using IVF. Since IVF is less invasive than laparoscopy and has a very high success rate, some couples will opt to skip laparoscopy and proceed directly to IVF. Even if a woman has severe adhesions that are not treated, this would not impact on her ability to conceive a pregnancy with IVF.

\section{Diagnostic Laparoscopy}

Laparoscopy can help physicians diagnose many gynecological problems including endometriosis, uterine fibroids and other structural abnormalities, ovarian cysts, adhesions (scar tissue), and ectopic pregnancy.

\section{Operative Laparoscopy}

During operative laparoscopy, many abdominal disorders can be treated safely through the laparoscope at the same time that the diagnosis is made. When performing operative laparoscopy, the physician inserts additional instruments such as probes, scissors, grasping instruments, biopsy forceps, electrosurgical or laser instruments, and suture materials through two or three additional incisions. Lasers, while a significant help in certain operations, are expensive and are not necessarily better or more effective than other surgical techniques used during operative laparoscopy.

\section{Robotic Assisted Laparoscopy}

Robotic assisted laparoscopic surgery (RAL) is a more recent development and a form of operative laparoscopy. 
In RAL, the instruments and telescope are very similar to conventional laparoscopy, but they are attached to a robot which in turn is controlled by the surgeon who is seated at a viewing console. This viewing console is usually located next to the patient, although the feasibility of a surgeon operating on a patient in another city or continent has been clearly demonstrated.

\section{Endometriosis and Infertility}

Women who have been diagnosed with endometriosis are more likely to experience infertility, and observational studies have shown that the monthly probability of pregnancy in women with endometriosis is about half of the probability in normal women. In spite of this welldocumented association, a true cause and effect relationship has not been established.

When women are having trouble conceiving, physicians must first determine whether an endometriosis diagnosis, which can only be determined via surgery, is necessary. When the woman is under 35 , has significant pain with her periods or with intercourse, and her partner has a normal semen analysis, a laparoscopy may be advised.

\section{Discussion}

Advances in imaging techniques have enabled accurate diagnosis of uterine and adnexal disease, thus redefining the role of laparoscopy. These techniques include two and three dimensional ultrasound, saline infusion sonography, computerized tomography (CT), and magnetic resonance imaging (MRI). Hysterosalpingography (HSG) and hysterosalpingo-contrastsonography (HyCoSy) are inexpensive, fast, and welltolerated methods of determining tubal patency, though their value when compared to laparoscopy is still a matter of debate ${ }^{21}$. These techniques have the advantage of being relatively noninvasive and less expensive compared to laparoscopy.

Diagnostic laparoscopy, which is often combined with hysteroscopy, is useful in ruling out Müllerian anomalies, revealing pelvic pathology, and assessing tubal function. In this era of improved imaging, the role of diagnostic laparoscopy, which is more invasive and expensive, has been questioned. This is especially so when initial clinical evaluation and imaging fail to find any abnormalities. The evidence for and against the use of laparoscopy in such cases has been inconsistent. In a retrospective study of 495 infertile women with unexplained infertility, laparoscopy before starting treatment revealed a significant incidence of abnormalities resulting in a changed treatment decision ${ }^{22}$ Among 172 patients (35\%) with abnormal findings, 21 (4\%) had severe abnormalities that resulted in a change of treatment to IVF or open surgery. In another 103 patients (21\%), abnormalities like endometriosis (stages I and II), and adhesions were directly treated by laparoscopic intervention. The laparoscopic yield was lower if surgery done to remove early stage endometriosis is omitted - 40 out of 495 cases (8.1\%). In a retrospective study of ovulation stimulation in 92 women, significant pelvic pathology was found in onethird of the patients failing to conceive after four ovulatory cycles of clomiphene citrate ${ }^{23}$ They concluded that laparoscopy may be omitted in women with normal HSG or suspected unilateral distal tubal pathology on HSG, since it was not shown to change the original treatment plan indicated by HSG in 95\% of the patients. They however recommended laparoscopy in cases with suspected bilateral tubal occlusion on HSG, since it altered the original treatment plan in 30\% of the patients ${ }^{24}$ al Badawi et al. retrospectively reviewed 265 women who had laparoscopies performed after normal hysterosalpingograms. Although 129 (49\%) had one or more abnormal laparoscopic findings, only $7 \%$ of cases had findings that might require standard operative laparoscopy or laparotomy, although not all were causally related to infertility. They advocated a micro-laparoscopic approach for women where history and HSG were not suggestive of pelvic disease, reserving conventional laparoscopy for those with suspected pathology on HSG. They went on to suggest bypassing laparoscopy in favor of assisted reproduction in such selected cases as the perceived benefit of surgical intervention is small 25

Only 4.6\% of laparoscopies performed for infertility over an 8-year study period satisfied these criteria. Although the vast majority of patients had some positive findings at laparoscopy $(87.4 \%, \mathrm{n}=111)$, it would be prudent to select those cases which would have findings significant enough to impair fertility and/or appreciably change further management. Capelo et al. defined 'positive laparoscopy' as surgical findings consisting of stages III or IV endometriosis, an endometrioma, pelvic adhesions, or tubal disease ${ }^{26}$ In this study, $26.8 \%$ of cases $(n=34)$ had a 'positive' laparoscopic finding. 
This included two uncommon diagnoses, viz. tuberculosis and adenocarcinoma arising from endometriosis.

There were no positive findings of hysteroscopy. This is not surprising as other authors have also demonstrated that a regular myometrial-endometrial interface and homogeneous endometrial structure on transvaginal sonography indicated a normal endometrium and precluded the need for diagnostic hysteroscopy 27

The benefit of laparoscopy in those women without 'positive findings', but with other minor pelvic pathology, is more contentious. Such cases accounted for $60.6 \%$ of the study population and largely included early stage endometriosis. A recent study showed that the likelihood of pregnancy was significantly reduced in infertile women with minimal or mild endometriosis compared with those infertile women with a normal pelvis. 28 Improved fecundity was seen with laparoscopic resection or ablation of minimal and mild endometriosis in a study of 341 infertile women, 29 though a smaller study involving 96 women did not show any benefit. ${ }^{30}$ In addition to endometriosis, laparoscopy is also useful in releasing adhesions, especially peritubal ones which might impair ovum transport due to decreased tubal motility. Restoring normal anatomy might increase pregnancy rates, although the existing studies are nonrandomized 31,32

Demonstration of effectiveness of laparoscopy would be incomplete without it being cost effective. Unfortunately, there are insufficient studies to assess the cost-benefit ratio of laparoscopy in unexplained infertility. The Practice Committee of the American Society of Reproductive Medicine suggests that laparoscopy should be seriously considered before applying aggressive empirical treatments involving significant cost and/or potential risks ${ }^{33}$ In a costeffectiveness analysis, a computer-generated decision analysis tree was used to compare expectant management, standard infertility treatment, and laparoscopy with and without infertility treatment. The study concluded that laparoscopy followed by expectant management is cost effective in the management of young couples with otherwise unexplained infertility

The timing of laparoscopy too has been a matter of debate. Although laparoscopy prior to initiating treatment looks attractive, the cost of this surgical procedure is high, especially when patients have to pay for the costs. Many clinicians thus prefer to treat couples with unexplained infertility with a few cycles of ovulation stimulation with IUI before proceeding to laparoscopy. A prospective randomized reallocation study to investigate the timing of laparoscopy after a normal hysterosalpingography was performed. This study, however, showed no significant difference in the prevalence of abnormalities with clinical consequences at laparoscopy before IUI when compared to laparoscopy after six cycles of IUI. The data suggested that the impact of the detection and the laparoscopic treatment of observed pelvic pathology prior to IUI seem negligible in terms of IUI outcome. The authors seriously questioned the value of routinely performing a diagnostic and/or therapeutic laparoscopy prior to IUI treatment ${ }^{34}$

\section{Conclusion}

Laparoscopy is used world-wide to investigate infertility. It is an essential part of full assessment and treatment of infertility ${ }^{2,6}$. It provides direct visualization of the pelvic organs, ovarian and tubal status and can elucidate the site of tubal obstruction

It has got an advantage of direct visualization of the pelvic organs and the peri-tubal status resulting in greater information as compared to hysterosalpingography and ultrasonography ${ }^{8}$. The advance in instrument technology has made this procedure more productive and less hazardous. Laparoscopy is the most dependable tool to investigate pelvic pathology

The role of laparoscopy in diagnosis of infertility both primary as well as secondary is established beyond any doubt.

\section{References}

1. Jose- Miller AB, Boyden JW, Frey KA. Infertility Am Fam Physician 2007; 75: 849.

2. Howkins and Bourne. The pathology of conception. In Shaw's textbook of Gynaecology 13th edition. Elsevier 2004.

3. Naz T. et al.JCPSP 2009; 19: 704.

4. Mehmood S. An audit of diagnostic laparoscopies for infertility. J Surg Pak 2003; 8: 8.

5. Tanahatoe SJ,Hompes PG, Lambalk CB. Investigation of the Infertile couple: should diagnostic laparoscopy be performedin the infertility workup programme in patients undergoing intrauterine insemination? Hum Reprod 2003; 18:8. 
6. Komori S, Fukuda Y, Horiuchi I,Tanaka H, Kasumi H, Shigeta M, et al . Diagnostic laparoscopy in infertility: a retrospective study. J Laparoendose Adv Surg Tech A 2003; 13:147-51.

7. TanahatoeSJ, Hompes PGA and Lambalk CB. Accuracy of diagnostic laparoscopy in the infertility work-up before intrauterine insemination. Fertil Steril. 2003b;79,361-66.

8. DonnezJ, Langerock S, Lecart C and Thomas K. Incidence of pathological factors not revealed by hysterosalpingography but disclosed by laparoscopy in 500 infertile women. Eur J Obstet Gynecol Reprod Biol. 1982;13, 369-75.

9. MusichJR and Behrman SJ. Infertility laparoscopy in perspective: review of five hundred cases. Am J Obstet Gynecol 1982;143,293-303.

10. CorsonSL, Cheng A and Gutmann JN. Laparoscopy in the 'normal' infertile patient: a question revisited. J Am Assoc Gynecol Laparosc 2000;7,317-24.

11. MolBW, Dijkman B, Wertheim P, Lijmer J, van der Veen $\mathrm{F}$ and Bossuyt PM. The accuracy of serum chlamydial antibodies in the diagnosis of tubal pathology: a metaanalysis. Fertil Steril. 1997;67,1031-37.

12. SwartP, Mol BW, van der Veen F, van Beurden M, Redekop WK and Bossuyt PM. The accuracy of hysterosalpingography in the diagnosis of tubal pathology: a meta-analysis. Fertil Steril 1995;64,486-91.

13. Wood GP. Laparoscopic examination of the normal infertile woman. Obstet Gynecol 1983; 62,642-43.

14. HenigI, Prough SG, Cheatwood M and DeLong E. Hysterosalpingography, laparoscopy and hysteroscopy in infertility. A comparative study. J Reprod Med. 1991;36,573-75.

15. OpsahlMS, Miller B and Klein TA. The predictive value of hysterosalpingography for tubal and peritoneal infertility factors. Fertil Steril. 1993;60,444-48.

16. ChaffkinLM, Nulsen JC, Luciano AA and Metzger DA. A comparative analysis of the cycle fecundity rates associated with combined human menopausal gonadotropin (hMG) and intrauterine insemination (IUI) versus either hMG or IUI alone. Fertil Steril. 1991;55, 252-57.

17. BelisleS, Collins JA, Burrows EA and Willan AR The value of laparoscopy among infertile women with tubal patency. J Soc Obstet Gynecol Can.1996;18,326-36.

18. al BadawiIA, Fluker MR and Bebbington MW. Diagnostic laparoscopy in infertile women with normal hysterosalpingograms. J Reprod Med. 1999;44,953-57.

19. RowePJ, Comhaire FH, Hargreave TB and Mellows HJ. WHO manual for the standardized investigation and diagnosis of the infertile couple. 1993

20. MarcouxS, Maheux R and Berube S. Laparoscopic surgery in infertile women with minimal or mild endometriosis. Canadian Collaborative Group on Endometriosis [see comments]. N Engl J Med. 1997;337,217-22.
21. Helmerhorst FM, Oei SG, Bloemenkamp KW, Keirse MJ. Consistency and variation in fertility investigations in Europe. Hum Reprod. 1995;10:2027-30. [PubMed]

22. Tanahatoe S, Hompes PG, Lambalk CB. Accuracy of diagnostic laparoscopy in the infertility work-up before intrauterine insemination. Fertil Steril. 2003;79:361-66. [PubMed]

23. Tanahatoe SJ, Hompes PG, Lambalk CB. Investigation of the infertile couple: Should diagnostic laparoscopy be performed in the infertility work up programme in patients undergoing intrauterine insemination? Hum Reprod. 2003;18:8. [PubMed]

24. Capelo FO, Kumar A, Steinkampf MP, Azziz R. Laparoscopic evaluation following failure to achieve pregnancy after ovulation stimulation with clomiphene citrate. Fertil Steril. 2003;80:1450-53. [PubMed]

25. Lavy Y, Lev-Sagie A, Holtzer H, Revel A, Hurwitz A. Should laparoscopy be a mandatory component of the infertility evaluation in infertile women with normal hysterosalpingogram or suspected unilateral distal tubal pathology? Eur J Obstet Gynecol Reprod Biol. 2004;114:64-68. [PubMed]

26. Corson SL, Cheng A, Gutmann JN. Diagnostic laparoscopy in infertile women with normal hysterosalpingograms. J Reprod Med. 1999;44:953-57. [PubMed]

27. Shalev J, Meizner I, Bar-Hava I, Dicker D, Mashiach R, Ben-Rafael Z. Predictive value of transvaginal sonography performed before routine diagnostic hysteroscopy for evaluation of infertility. Fertil Steril. 2000;73:412-17. [PubMed]

28. Akande VA, Hunt LP, Cahill DJ, Jenkins JM. Differences in time to natural conception between women with unexplained infertility and infertile women with minor endometriosis. Hum Reprod. 2004;19:96. [PubMed]

29. Marcoux S, Maheux R, Berube S. Laparoscopic surgery in infertile women with minimal or mild endometriosis: Canadian Collaborative Group on Endometriosis. N Engl J Med. 1997;337:217-22. [PubMed]

30. Parazzini F. Ablation of lesions or no treatment in minimal-mild endometriosis in infertile women: A randomized trial. Hum Reprod. 1999;14:1332-34. [PubMed]

31. Tulandi T, Collins JA, Burrows E, Jarrell JF, McInnes RA, Wrixon W, et al. Treatment-dependent and treatment independent pregnancy among women with periadnexal adhesions. Am J Obstet Gynecol. 1990;162:354-57. [PubMed]

32. Saravelos HG, Li TC, Cooke ID. An analysis of the outcome of microsurgical and laparoscopic adhesiolysis for chronic pelvic pain. Hum Reprod. 1995;10:2887-94. [PubMed]

33. Practice Committee of the American Society of Reproductive Medicine. Optimal evaluation of the infertile female. Fertil Steril. 2006; 86:S264-67. [PubMed]

34. Tanahatoe SJ, Lambalk CB, Hompes PG. The role of laparoscopy in intrauterine insemination: A prospective randomized reallocation study. Hum Reprod. 2005; 20:3225-30. [PubMed] 